\title{
Study of dielectric relaxation behavior in $\mathrm{Nd}_{2} \mathrm{CuO}_{4}$
}

\author{
J.W. Chen *, J.C. Wang, Y.F. Chen \\ Department of Physics, National Taiwan University, Taipei, Taiwan, ROC
}

Received 25 February 1997; accepted 1 April 1997

\begin{abstract}
The dielectric properties of the helium reduced $\mathrm{Nd}_{2} \mathrm{CuO}_{4}$ samples were studied by means of capacitance $C$ and dissipation factor $\tan \delta$ measurements with the test frequency $f$ in the range from $20 \mathrm{~Hz}$ to $1 \mathrm{MHz}$ and at temperature $T$ between $5 \mathrm{~K}$ and $325 \mathrm{~K}$. In addition to obtain high dielectric constant at room temperature and low frequencies, two frequency-dependent peaks in the $\tan \delta$ curves and corresponding features in the real part dielectric constant $\varepsilon^{\prime}$ curves were observed near $118 \mathrm{~K}$ and $255 \mathrm{~K}$ for $f=100 \mathrm{~Hz}$. The peak temperatures observed in the tan $\delta$ curves at various test frequency $f$ follow the relation $f=f_{\mathrm{c}} \mathrm{e}^{-W / k T}$, with $f_{\mathrm{c}}=3.1 \times 10^{13}\left(2.8 \times 10^{14}\right) \mathrm{Hz}$ and the activation energy $W=0.282$ $(0.60) \mathrm{eV}$ for the $118 \mathrm{~K}(255 \mathrm{~K})$ peak for the sample annealed at $880^{\circ} \mathrm{C}$. The low temperature activation is attributed to the excitations from the ground state of ${ }^{4} I_{9 / 2}$ multiplets to the first excited states of the ${ }^{4} I_{11 / 2}$ multiplets. This assignment is further confirmed by the fact that the activation energy is reduced to $0.245 \mathrm{eV}$ for the sample reduced at $920^{\circ} \mathrm{C}$. The high temperature activation is related to the intrinsic excitations of the $\mathrm{CuO}_{2}$ layers, and its activation energy is unaffected by the reduction temperatures used in this study. Our results indicate that reducing treatment produces the effect of increasing oxygen vacancies, probably through the removal of the $\mathrm{O}(2)$ 's (i.e., oxygens inbetween the $\mathrm{Nd}$ layers) in $\mathrm{Nd}_{2} \mathrm{CuO}_{4}$. $\mathcal{C} 1997$ Elsevier Science B.V.
\end{abstract}

PACS: $77.22 \mathrm{Gm} ; 77.84 \mathrm{Bw}$

Keywords: Dielectric

$\mathrm{Nd}_{2} \mathrm{CuO}_{4}$ has attracted a lot of research interest since the discovery of the electron-doped superconductivity in $R_{2-x} \mathrm{Ce}_{x} \mathrm{CuO}_{4-y}(R=\mathrm{Pr}, \mathrm{Nd}, \mathrm{Sm}$, and Eu) $[1,2]$ and $R_{2-x} \mathrm{Th}_{x} \mathrm{CuO}_{4-y}(R=\mathrm{Pr}, \mathrm{Nd}$, and $\mathrm{Sm})[2-4]$ with a superconducting transition temperature up to $24 \mathrm{~K}$. This compound exhibits several magnetic transitions below room temperature in association with the antiferromagnetic ordering of the

\footnotetext{
* Corresponding author. Tel.: +8862362 6937; fax: +8862 363 9984; e-mail:jwchen@phys.ntu.edu.tw.
}

$\mathrm{Cu}$ spins [5-9] or the $\mathrm{Nd}^{3+}$ ions [10-12]. In addition, magnetic susceptibility studies on single crystals of $\mathrm{Nd}_{2} \mathrm{CuO}_{4-y}$, reveal anisotropic characteristic with the easy axis of magnetization parallel to the basel plane below $T \approx 100 \mathrm{~K}[13,14]$. Although the above mentioned magnetic transition temperatures in $\mathrm{Nd}_{2} \mathrm{CuO}_{4-,}$, are not related to the oxygen deficiency in the samples [9,11], it has been shown that reduce treatment does reveal pronounced effects in the room temperature resistivity, optical reflectivity and transmission spectra [15], Raman spectra [16], and inelastic neutron scattering [17] data. Unlike other high- $T_{0}$ 
oxides, the oxygen stoichiometry and the effect of reduce treatment remain controversial $[18-22]$ for $\mathrm{Nd}_{2} \mathrm{CuO}_{4-y}$. Recently, Jandl et al. [23] studied the intermultiplet crystal-field excitations in $\mathrm{Nd}_{2} \mathrm{CuO}_{4-y}$ extensively using the Raman scattering and conjectured that the effect of reduction is to remove the extra apical oxygens $O(3)$ 's in the sample. According to their result, reduce treatment will decrease the amount of the carrier concentration in the sample and increases the resistivity instead of decrease the room temperature resistivity by one order of magnitude [15]. Therefore, a comparison of the transport behaviors for $\mathrm{Nd}_{2} \mathrm{CuO}_{4-y}$ under different treatment conditions is still necessary.

Recently, measurements of the dielectric properties of the insulating phases of $\mathrm{YBa}_{2} \mathrm{Cu}_{3} \mathrm{O}_{6+\delta}$ [24,25], $\mathrm{PrBa}_{2} \mathrm{Cu}_{3} \mathrm{O}_{7}$ [26], and $\mathrm{La}_{2} \mathrm{CuO}_{4}$ [27] revealed a high dielectric constant $\left(\varepsilon^{\prime}>10^{3}\right)$ at room temperature and low frequencies. This ferroelectriclike polarization is closely related to the antiferromagnetic ordering of the $\mathrm{Cu}$ moments within the $\mathrm{Cu}-\mathrm{O}$ planes and chains of the samples and was suggested to be a common feature of the high- $T_{c}$ oxides. Since $\mathrm{Nd}_{2} \mathrm{CuO}_{4}$ also contains $\mathrm{CuO}_{2}$ layers and the $\mathrm{Cu}$ spins order antiferromagnetically at $T_{\mathrm{N}} \approx$ $250 \mathrm{~K} \mathrm{[5-9]}$. It is interesting to see whether such ferroelectric like behavior also exists in this system. Moreover, since the dielectric properties are very sensitive to the local electric field distribution within the samples, studies of the temperature and frequency dependences of the dielectric behaviors of $\mathrm{Nd}_{2} \mathrm{CuO}_{4}$ can reveal useful information about the defect concentration and provide us with better insight about the effect of reduce treatment in this compound. Such information will be useful for understanding why it is necessary for reduce treatment to produce superconductivity in $\mathrm{Nd}_{1.85} \mathrm{Ce}_{0.15} \mathrm{CuO}_{4-y}$. We have studied the dielectric properties on the He-reduced $\mathrm{Nd}_{2} \mathrm{CuO}_{4-y}$, samples in temperatures between $5 \mathrm{~K}$ and $325 \mathrm{~K}$ with test frequencies in the range from $20 \mathrm{~Hz}$ to $1 \mathrm{MHz}$.

The samples used in this study were prepared by solid-state reaction from high purity $\mathrm{Nd}_{2} \mathrm{O}_{3}$ and $\mathrm{CuO}$ powders. Stoichiometric mixtures of these powders were ground thoroughly and heated in air at $900^{\circ} \mathrm{C}$ for 1 day and furnace cooled to room temperature. The resulting powders were pressed into pellets and fired in air at $900^{\circ} \mathrm{C}$ for another day and cooled to room temperature. According to Tarascon et al. [10], reduce treatment above $900^{\circ} \mathrm{C}$ is essential for the occurrence of superconductivity and complete oxygen removal in $\mathrm{Nd}_{2-x} \mathrm{Ce}_{x} \mathrm{CuO}_{4-y}$. Because we are interested in the effect of reduce treatment on the removal of the oxygens, we sintered the pellets in flowing $\mathrm{He}$ at $880^{\circ} \mathrm{C}$ and $920^{\circ} \mathrm{C}$, respectively, for comparison in this study. Powder X-ray diffraction data reveal no impurity phase in the samples. Because the reported amount of oxygen removal is small $(0.01-0.04$ per unit cell) and the possibility of oxygen inhomogeneity in this compound, we did not make an effort to determine the oxygen content of the samples. The capacitance $C$ and dissipation factor $\tan \delta$ measurements were performed on the diskshaped pellets with silver paint coated on two sides of the pellets as the electrodes using a HP $4284 \mathrm{~A}$ LCR meter in a He-dewar with a Si-diode as the temperature thermometer. The radius $R$ of the pellets is $0.5 \mathrm{~cm}$ and the thickness $d$ is of $0.1 \mathrm{~cm}$. The real part dielectric constant $\varepsilon^{\prime}$ can be determined from the value of $C$ through $\varepsilon^{\prime}=C / C_{0}$ where $C_{0}$ is $\varepsilon_{0} A b / d$ with $A$ being the area of the surface of the disk $\left(A=\pi R^{2}\right)$ and $b$ being the correction factor which has a value of 1.286 in our case.

The real part dielectric constant $\varepsilon^{\prime}$ as a function of temperature $T$ curves with selected test frequencies $f$ for $\mathrm{Nd}_{2} \mathrm{CuO}_{4}$ annealed at $T_{\mathrm{a}}=920^{\circ} \mathrm{C}$ are plotted in Fig. 1(a). These $\varepsilon^{\prime}$ curves show distinct behaviors in different temperature regions. At the lowest temperatures, $\varepsilon^{\prime}$ is almost temperature and frequency independent and approaches a value of $\approx 8.4$ at $T=4 \mathrm{~K}$. With increasing temperature $T, \varepsilon^{\prime}$ increases and frequency dispersion develops. For $T \leq 60 \mathrm{~K}, \varepsilon^{\prime}$ exhibits a behavior characteristic of normal dielectrics and increases slightly with increasing $T$. The effect of test frequency on the $\varepsilon^{\prime}$ curves is weak in this temperature regime. For $T \geq$ $120 \mathrm{~K}$, the effects of temperature and frequency on $\varepsilon^{\prime}$ become more pronounced and $\varepsilon^{\prime}$ starts to increase rapidly with increasing $T$ and decreases dramatically as the test frequency increases. Finally, an inflection in $\varepsilon^{\prime}$ appears at approximately $255 \mathrm{~K}$, near the temperature $T_{\mathrm{N}}$ where antiferromagnetic ordering occurs in this system. The obtained value of $\varepsilon^{\prime}$ at room temperature is about $10^{4}$, a value that is comparable to that have been reported for other high- $T_{\mathrm{c}}$ oxides [24-27]. The $\varepsilon^{\prime}$ curves for the sam- 
ple annealed at $880^{\circ} \mathrm{C}$, with the same selected test frequencies, are plotted in Fig. 1(b). Although similar temperature and frequency characteristics were observed, it is interesting to notice that the effects of temperature and test frequency on $\varepsilon^{\prime}$ are even weaker for $T \leq 60 \mathrm{~K}$ and the value of $\varepsilon^{\prime}$ at room temperature is smaller.

The fact that $\varepsilon^{\prime}$ becomes independent of frequency at lowest temperatures indicates that the observed response represents the static dielectric response of this system in the absence of dipolar effects. The slight increase of $\varepsilon^{\prime}$ with increasing temperature for $T \leq 60 \mathrm{~K}$ may result primarily from the increase in the lattice polarizability or softening
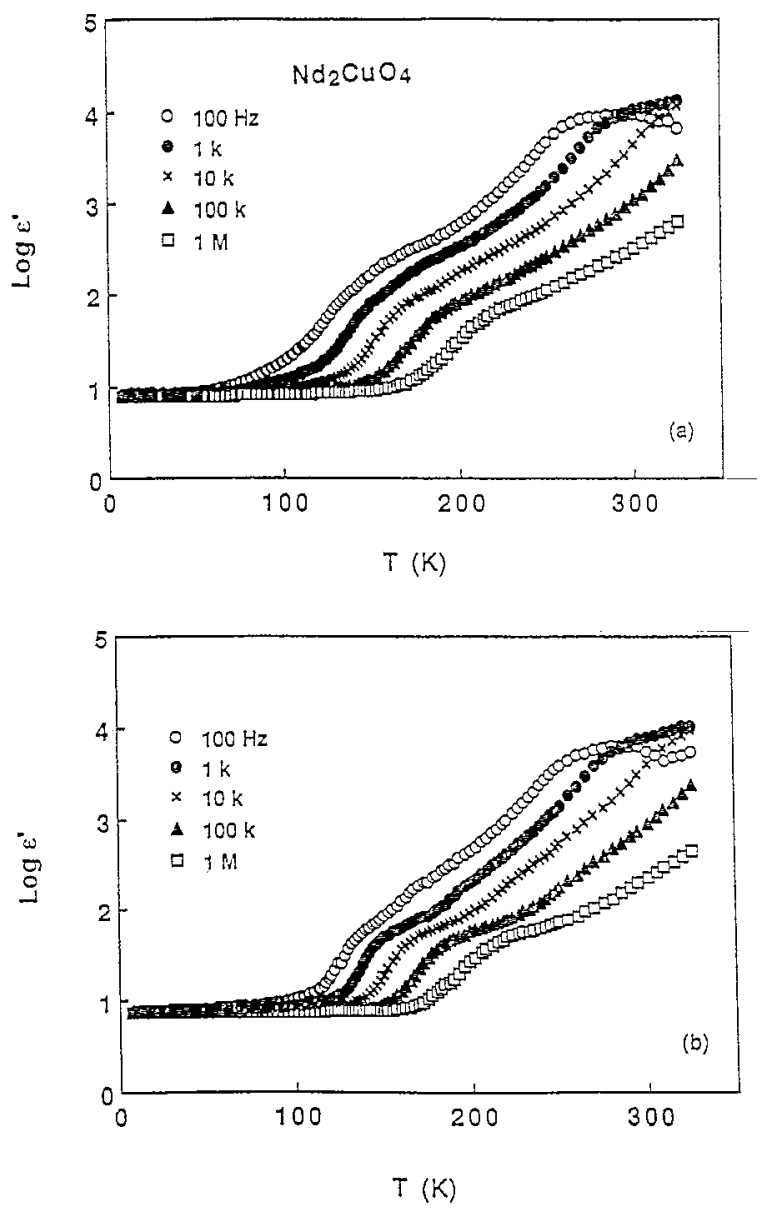

Fig. 1. The logarithm of the real part dielectric constant $\log \varepsilon^{\prime}$ as a function of temperature $T$ curves with selected test frequencies for the $\mathrm{Nd}_{2} \mathrm{CuO}_{4}$ sample annealed at (a) $920^{\circ} \mathrm{C}$ and (b) $880^{\circ} \mathrm{C}$.
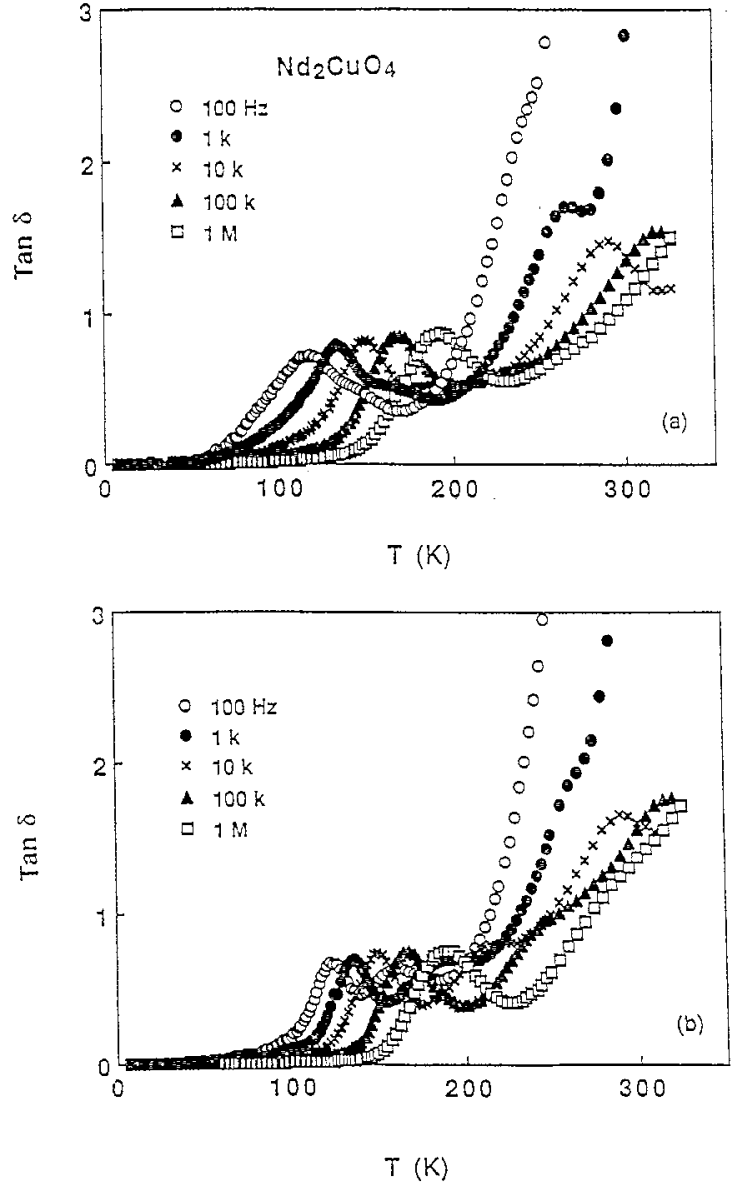

Fig. 2. The dissipation factor $\tan \delta$ versus temperature $T$ curves with selected test frequencies for the $\mathrm{Nd}_{2} \mathrm{CuO}_{4}$ sample annealed at (a) $920^{\circ} \mathrm{C}$ and (b) $880^{\circ} \mathrm{C}$.

of the lattice with increasing $T$. The rapid increase of $\varepsilon^{\prime}$ with increasing $T$ and strong frequency dispersion in $\varepsilon^{\prime}$ observed for $T \geq 120 \mathrm{~K}$ reveals characteristic of dipolar effects that are controlled by the activation of defects or localized charge centers in the samples. Such localized charge carriers are believed to be prevalent in the insulating phases of the high- $T_{\mathfrak{c}}$ oxides. The smaller value of $\varepsilon^{\prime}$ observed at room temperature for the sample annealed with lower $T_{\mathrm{a}}$ indicates that the carrier concentration is smaller for that sample.

Shown in Fig. 2(a) are the dissipation factor $\tan \delta$ versus temperature $T$ curves for selected values of $f$ for the sample annealed with $T_{\mathrm{a}}=920^{\circ} \mathrm{C}$. At low 
temperatures, the observed value of $\tan \delta$ is quite small $(<0.005)$ and $\tan \delta$ increases slightly with increasing $T$ for $T \leq 60 \mathrm{~K}$. Above $60 \mathrm{~K}$, $\tan \delta$ increases substantially with increasing $T$ with a peak occurring near $120 \mathrm{~K}$. This peak temperature $T_{0}$ increases monotonically with increasing test frequency from $118 \mathrm{~K}$ for $f=100 \mathrm{~Hz}$ to $\approx 193 \mathrm{~K}$ for $f=1 \mathrm{MHz}$. Above $120 \mathrm{~K}$, $\tan \delta$ exhibits characteristics similar to that of $\varepsilon^{\prime}$ and another peak occurs near $255 \mathrm{~K}$ for $f=100 \mathrm{~Hz}$. This peak temperature $T_{1}$ is closely related to the inflection temperatures observed in the $\varepsilon^{\prime}$ curves and also increases monotonically with $f$ to a value of $\approx 320 \mathrm{~K}$ for $f=100 \mathrm{kHz}$. Above $100 \mathrm{kHz}$, no maximum was observed in the $\tan \delta$ curves below the maximum temperature (325 K) measured. The rapid increase of $\tan \delta$ for $T^{\prime}>T_{1}$ indicates that dc effect becomes important above this temperature. For the sample annealed at $880^{\circ} \mathrm{C}$, the $\tan \delta$ curves (shown in Fig. 2(b)) exhibit characteristics similar to that of the $920^{\circ} \mathrm{C}$ annealed sample. However, the value of $T_{0}$ at $f=100 \mathrm{~Hz}$ is higher $(122.5 \mathrm{~K})$ and the effect of test frequency on $T_{0}$ becomes weaker $\left(T_{0}=190 \mathrm{~K}\right.$ for $\left.f=1 \mathrm{MHz}\right)$ for the sample annealed at $880^{\circ} \mathrm{C}$. The extra pronounced frequency dependent features in the $\tan \delta$ curves observed between $T_{0}$ and $T_{1}$ for the sample annealed at $880^{\circ} \mathrm{C}$ may indicate oxygen inhomogeneity in that sample.

For a Debye-type dielectric relaxation process the dissipation factor $\tan \delta=\chi^{\prime \prime}(\omega) /\left(1+\chi^{\prime}(\omega)\right)=(2$ $\left.-\omega^{2} \tau^{2}\right) /\left(2+\omega^{2} \tau^{2}\right.$ ) (where $\chi^{\prime}(\omega)$ and $\chi^{\prime \prime}(\omega)$ are the real part and imaginary part of the dielectric susceptibility, respectively) and exhibits a peak for $\omega \tau=\sqrt{2}$. In order to see the temperature dependence of the peak position, the logarithm of the test frequency $\ln f$ versus the reciprocal of the peak temperatures ( $T_{0}$ values and $T_{1}$ values) at the corresponding test frequency, $1000 / T$, are plotted in Fig. 3(a) and (b). The observed linear relations between $\ln f$ and $1000 / T$ for both peak temperatures indicate that the relaxation behaviors are thermally activated. From the deduced relation $f=f_{\mathrm{C}} \mathrm{e}^{-W / k_{\mathrm{B}} T}$, we obtain $f_{\mathrm{c}}=3.1 \times 10^{13}\left(2.1 \times 10^{12}\right) \mathrm{Hz}$ and the activation energy $W=0.282(0.245) \mathrm{eV}$ for the low temperature peaks ( $T_{0}$ values) for the sample annealed at $880^{\circ} \mathrm{C}\left(920^{\circ} \mathrm{C}\right)$, respectively. For the high temperature peaks $\left(T_{1}\right.$ values), both $f_{\mathrm{c}}$ and the activation energy $W$ are not affected by the annealing tempera-
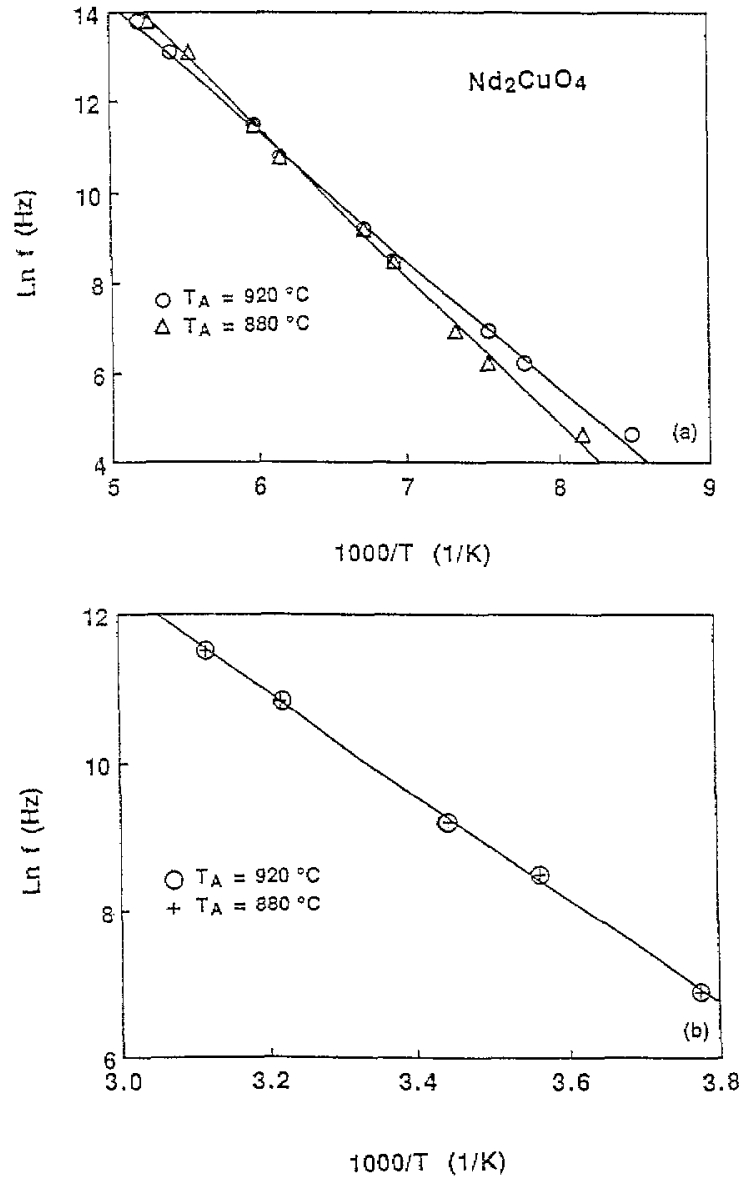

Fig. 3. The natural logarithm of the test frequency $\ln f$ versus the reciprocal of the peak temperatures in the $\tan \delta$ curves ( $T_{0}$ values for (a) and $T_{1}$ values for (b)) at the corresponding test frequency, $1000 / T$, for the $\mathrm{Nd}_{2} \mathrm{CuO}_{4}$ samples annealed at $920^{\circ} \mathrm{C}$ and $880^{\circ} \mathrm{C}$. The solid lines are least square fits for the data.

tures and we have $f_{\mathrm{c}}=2.8 \times 10^{14} \mathrm{~Hz}$ and $W=0.60$ $\mathrm{eV}$ for both samples. Comparing with the existing data, it is interesting that our values of $W$ for the low temperature peaks are related to that of the low energy peaks in the mid-infrared data [28] and are close to the two peaks' values $(0.247 \mathrm{eV}$ and 0.298 $\mathrm{eV)}$ as revealed from the inelastic-neutron-scattering [17] and Raman scattering [23] data for the excitations from the ground state of ${ }^{4} \mathrm{I}_{9 / 2}$ multiplets to the first excited states of the ${ }^{4} \mathrm{I}_{11 / 2}$ multiplets in $\mathrm{Nd}_{2} \mathrm{CuO}_{4}$. Therefore, we believe that the excitation for the low temperature peaks is of the same origin. 
Moreover, the lower value of the activation energy $W$ for the sample with higher annealing temperature $T_{a}$ indicates that it is the oxygen deficiency that is responsible for the relaxation behavior observed here. Since with higher reduction temperature, more oxygen vacancies will be removed from the sample which in turn produces more carriers in the sample that occupy the excited levels of the ground state multiplets. As a result, we have an excitation energy for the sample annealed at $920^{\circ} \mathrm{C}$ corresponding to the lower value $(0.247 \mathrm{eV})$ of the doublet as observed from the inelastic-neutron-scattering and $\mathrm{Ra}-$ man scattering data. The smaller value of $W$ (compared with $0.298 \mathrm{eV}$ ) for the sample annealed with $T_{\mathrm{a}}=880^{\circ} \mathrm{C}$ indicates that the oxygen removal is not complete at that temperature.

Mid-infrared absorption data for the undoped lamellar copper oxides revealed an universal optical excitation near $0.5 \mathrm{eV}$ which indicates that this absorption is from the intrinsic excitations of the $\mathrm{CuO}_{2}$ layers [28]. From the obtained value of $W$ $(0.60 \mathrm{eV})$ and the peak temperature in the $\tan \delta$ curve (for $f=100 \mathrm{~Hz}$ ), we believe that the excitation for the high temperature peaks is from the $\mathrm{CuO}_{2}$ layers. Since reduce treatment at different temperatures $\left(880^{\circ} \mathrm{C}\right.$ and $\left.920^{\circ} \mathrm{C}\right)$ produces no effect on the value of $W$, it is reasonable to conjecture that the oxygen vacancies are not in the $\mathrm{CuO}_{2}$ layers (i.e., $O(1)$ as shown in Ref. [23]). Instead, it is probably the oxygens inbetween the Nd layers (i.e., O(2)'s) that were removed after reduction.

In summary, we have studied the temperature and frequency dependences of the dielectric behaviors of $\mathrm{Nd}_{2} \mathrm{CuO}_{4}$ samples reduced at different temperatures. In addition to obtain high dielectric constant at room temperature and low frequencies, we also observed two thermally activated relaxations near $118 \mathrm{~K}$ and $255 \mathrm{~K}$ with the activation energy $W$ of $0.282(0.245)$ $\mathrm{eV}$ and $0.60 \mathrm{eV}$, respectively. The low temperature activation corresponds to the excitations from the ground state of ${ }^{4} \mathrm{I}_{9 / 2}$ multiplets to the first excited states of the ${ }^{4} \mathrm{I}_{11 / 2}$ multiplets and $W$ is reduced with higher reduction temperature. The high temperature activation is related to the intrinsic excitations of the $\mathrm{CuO}_{2}$ layers, and its activation energy is unaffected by the reduction temperatures used in this study. From this, we conjecture that reducing treatment produces the effect of increasing oxygen vacancies, probably through the removal of the oxygens inbetween the $\mathrm{Nd}$ layers in $\mathrm{Nd}_{2} \mathrm{CuO}_{4}$

\section{Acknowledgements}

This work is supported by the ROC National Science Council under Grant No. NSC85-2112-M002-006.

\section{References}

[1] T. Tokura, H. Tagaki, S. Uchida, Nature 337 (1989) 345.

[2] J.T. Markert, E.A. Early, T. Bionholm, S. Ghamaty, B.W. Lee, J.J, Neumeiert, R.D. Price, M.B. Maple, Physica C 158 (1989) 178

[3] J.T. Markert, M.B. Maple, Solid State Commun. 70 (1989) 145.

[4] E.A. Early, N.Y. Ayoub, J, Beille, J.T. Markert, M.B. Maple, Physica C 160 (1989) 320.

[5] R. Saez Puche, M. Norton, W.S. Glaunsinger, Mater. Res, Bull. 17 (1982) 1523.

[6] R. Saez Puche, M. Norton, T.R. White, W.S. Glaunsinger, J, Solid State Chem. 50 (1983) 281.

[7] G.M. Luke, B.J. Sternlieb, Y.J. Uemara, J.H. Brewer, R. Kadono, R.F. Kiefl, S.R. Kreitzman, T.M. Riseman, J. Gopalakrishman, A.W. Sleight, M.A. Subramanian, S. Uchida, H. Takagi, Y. Tokura, Nature 338 (1989) 49.

[8] S. Skanthkumar, H. Zhang, T.W. Clinton, W.-H. Li, J.W Lynn, Z, Fisk, S.-W Cheong, Physica C 160 (1989) 124.

[9] Y. Endoh, M. Matsuda, K. Yamada, K. Kakurai, Y. Hidaka, G. Shirane, R.J. Birgeneau, Phys. Rev. B 40 (1989) 7023.

[10] J.-M. Tarascon, E. Wang, L.H. Greene, B.G. Bagley, G.W. Hull, S.M. D'Edigo, P.F. Miceli, Z.Z. Wang, T.W. Jing, J. Clayhold, D. Rrawner, N.P. Ong, Phys. Rev. B 40 (1989) 4494.

[11] M.F. Hundley, J.D. Thompson, S.-W. Cheong, Z. Fisk, Physica C 158 (1989) 102

[12] C.L. Seaman, N.Y. Ayoub, T. Bjornholm, E.A. Early, S. Ghamaty, B.W. Lee, J.T. Markert, J.J. Neumeier, P.T. Tsai, M.B. Maple. Physica C 159 (1989) 391.

[13] S. Ghamaty, B.W. Lee, J.T. Markert, E.A. Early, T. Bjornholm, C.L. Seaman, M.B. Maple, Physica C 160 (1989) 217.

[14] J.W. Lynn, I.W. Sumarlin, S. Skanthakumar, W.-H. Lee, R.N. Shelton, J.L. Peng, Z. Fisk, S.-W. Cheong, Phys. Rev. B 41 (1990) 2569.

[15] K. Hirochi, S. Hayahsi, H. Adachi, T. Mitsuyu, T. Hirao, K. Setsune, K. Wasa, Physica C 160 (1989) 273.

[16] P. Dufoue, S. Jandl, C. Thomsen, M. Cardona, B.M. Wanklyn, C. Changkang, Phys. Rev. B 51 (1995) 1053.

[17] A.T. Boothroyd, S.M. Doyle, D.McK. Paul, R. Osborn, Phys. Rev. B 45 (1992) 10075.

[18] E. Muran, A.I. Nazzal, T.C. Huang, J.B. Torrance, Physica C 160 (1989) 30. 
[19] E. Wang, J.-M. Tarascon, L.H. Greene, G.W. Hull, W.R. McKinnon, Phys. Rev. B 41 (1990) 6582.

[20] P.G. Radaelli, J.D. Jorgensen, A.J. Schultz, J.L. Peng, R.L. Greene, Phys. Rev, B 49 (1994) 15322.

[21] K. Suzuki, K. Kishio, T. Hasegawa, K. Kitazawa, Physica C $166(1990) 357$.

[22] J.S. Kim, D.R. Gaskell, Physica C 209 (1993) 381.

[23] S. Jandl, P. Dufour, T. Strach, T. Ruf, M. Cardana, C. Chen, B.M. Wanklyn, Phys. Rev. B 52 (1995) 15558.

[24] G.A. Samara, W.F. Hammeter, E.L. Venturini, Phys. Rew, B $41(1990) 8974$
[25] C.M. Rey, H. Mathias, L.R. Testardi, Phys. Rev. B 45 (1992) 10639.

[26] D. Gao, J.W. O’Reilly, J.E. Crow, L.R. Testardi, Phys. Rey. B 47 (1993) 11510.

[27] G.P. Mazzara, S. Skirius, G. Gao, G. Chern, R.J. Clark, J.E. Crow, H. Mathias, J.W. O'Reilly, L.R. Testardi, Phys. Rev. B 47 (1993).

[28] J.D. Perkins, J.M. Graybeal, M.A. Kastner, R.J. Birgeneau, J.P. Falck, M. Greven, Phys. Rev. Lett. 71 (1993) 1621. 